Tropical Journal of Pharmaceutical Research April 2018; 17 (4): 709-713

ISSN: $1596-5996$ (print); 1596-9827 (electronic)

(C) Pharmacotherapy Group, Faculty of Pharmacy, University of Benin, Benin City, 300001 Nigeria.

\title{
A cross-sectional study of the actual use of eye drops in a Saudi community
}

\author{
Abdulsalam Alfawzan', Anas Al Ahmed ${ }^{2}$, Sara Almuhanna1, Hadeel Alfahmi \\ Abdulallah Alshein ${ }^{1}$, Mohammad Kariri ${ }^{1}$, Amani Alsultan ${ }^{1}$, Ahmad Almeman ${ }^{4 *}$ \\ ${ }^{1}$ Department of Pharmaceutical Care, Buraidah Central Hospital, Ministry of Health, Buraidah, ${ }^{2}$ Department of Pharmacy \\ Practice, College of Clinical Pharmacy, King Faisal University, Al Ahsa, ${ }^{3}$ Department of Clinical of Clinical Pharmacy, College of \\ Pharmacy, Umm-Al-Qura University, Makkah, ${ }^{4}$ Research Centre, College of Medicine, Qassim University, Qassim, Kingdom of \\ Saudi Arabia
}

*For correspondence: Email: meman@qu.edu.sa; Tel: +966-5488897

Sent for review: 16 August 2017

Revised accepted: 23 March 2018

\begin{abstract}
Purpose: To investigate the actual use of eye drops with respect to their administration, storage, and disposal in a community in Saudi Arabia.

Methods: A cross-sectional observational survey was conducted online. All the relevant data were collected using a standardized questionnaire. The questionnaire consisted of three sections: demographic characteristics of the participants, attitude of participants with respect to the administration and storage of eye drops, and the participants' understanding of the role of pharmacist in enhancing patients' compliance with eye drop regimens.

Results: A total of 720 participants were included in the study, viz, 229 males (31.8\%) and 491 females (68.2\%). Majority of the participants (82.5\%) indicated that they avoided touching their eyes with the bottle tip. Likewise, most participants $(90.8 \%)$ reported that they applied eye ointment after administering eye drops while $30.6 \%$ allowed an interval of $<5$ min between eye drop and ointment administration. However, $30.5 \%$ of the participants gave an interval of $5-10$ min between eye drop and ointment treatment. Furthermore, $32.5 \%$ reported that they consistently washed their hands before administering eye drops, while only $29.6 \%$ occasionally washed their hands.

Conclusion: These results indicate that all the patients used eye drops improperly at some point in time, and thus, there is a need for counselling on the appropriate use of eye drops as an essential step towards successful treatment.
\end{abstract}

Keywords: Eye drops, Ophthalmic preparations, Adherence, Instillation

\begin{abstract}
This is an Open Access article that uses a funding model which does not charge readers or their institutions for access and distributed under the terms of the Creative Commons Attribution License (http://creativecommons.org/licenses/by/4.0) and the Budapest Open Access Initiative (http://www.budapestopenaccessinitiative.org/read), which permit unrestricted use, distribution, and reproduction in any medium, provided the original work is properly credited.

Tropical Journal of Pharmaceutical Research is indexed by Science Citation Index (SciSearch), Scopus, International Pharmaceutical Abstract, Chemical Abstracts, Embase, Index Copernicus, EBSCO, African Index Medicus, JournalSeek, Journal Citation Reports/Science Edition, Directory of Open Access Journals (DOAJ), African Journal Online, Bioline International, Open-J-Gate and Pharmacy Abstracts
\end{abstract}

\section{INTRODUCTION}

The eye is a complex organ with unique anatomy and physiology. Consequently, ophthalmic drug delivery is one of the most interesting and challenging endeavors facing the pharmaceutical scientists [1,2]. Pharmaceutical companies produce lots of ophthalmic dosage forms. These products include ointments and eye drops which are used for treating diseases associated with ocular health. Eye drops constitute approximately $90 \%$ of the ophthalmic dosage forms widely 
used to manage acute and chronic ocular conditions $[3,4]$.

The use and administration of eye drops should be done properly to maximize their therapeutic effects, and to reduce adverse reactions [5]. In fact, poor adherence to ocular treatment regimens could lead to ocular discomfort, loss of vision, or even blindness [3]. Factors that may contribute to non- adherence to treatment regimens include the shape and size of eye drop bottle, ease of application, and number of prescriptions and complexity of the drug administration protocols [6].

Some studies aimed at investigating the actual use of eye drops have been carried out. A Canada-based study showed that $92.6 \%$ of patients with eye disorders used improper techniques in administrating eye drops [7]. In a study done in India on the awareness of patients about ideal techniques for the use and administration of eye drops, it was revealed that almost $30 \%$ of patients who use eye drops believe that there is no problem with "back to back" eye drop instillation [8]. Unfortunately, there are only very few publications on the actual use of eye drops in Kingdom of Saudi Arabia. The aim of this cross-sectional study was to examine the behavior of individuals with respect to administration, storage and disposal of eye drop preparations. In addition, the study investigated the role of pharmacists in enhancing patients' compliance with the use of eye drops.

\section{METHODS}

\section{Study design}

The data reported here came from a crosssectional study that made use of a questionnaire.

\section{Sample size}

The sample size was 643 randomly selected participants who live in Kingdom of Saudi Arabia and used eye drops previously.

\section{Inclusion criteria}

Patients using eye drops for any ophthalmic indications, are able to read Arabic, and accepted the survey conditions.

\section{Ethical approval}

This research was approved on November 17, 2016 by Regional Research Ethics Committee, registered at National Committee of Bio and Med Ethics Registration no. H-04-Q-001.

\section{Data collection}

Data were collected by using a standardized questionnaire (Appendix) which was distributed through social networking (Facebook and Twitter). The questionnaire was developed on the basis of other related studies and opinions from experiences. The questionnaire consisted of three sections. The first section contained questions on demographic information on participants, while the second section contained questions for assessing the behavior of individuals with respect to administration and storage of eye drops. The third and last section concerned questions aimed at investigating the role of pharmacists in enhancing patient compliance with the use of eye drops. Information about the use, administration and storage of eye drops were derived from literature $[9,10]$.

\section{Statistical analysis}

The data were computed and analyzed with SPSS software. Data description was reflected as frequency distribution. Responses were compared using Chi-square test. Statistical significance was assumed at $p \leq 0.05$.

\section{RESULTS}

\section{Demographic characteristics}

There were a total 720 study participants made up of 229 (31.8\%) males and 491 (68.2\%) females. More than half of the participants $(67.2 \%)$ were from central region of Kingdom of Saudi Arabia. More than half of the participants $(63.3 \%)$ were aged between 18 and 35 years, while more than two-thirds $(80.3 \%)$ of the participants were from college. The demographic characteristics of the participants are detailed in Table 1.

\section{Techniques used in the administration of eye drops}

Most of participants (82.5\%) indicated that they never touched their eye with bottle tip. In addition, almost all the participants (90.8\%) reported that they applied ointments after administering eye drop. Moreover, almost onethird of the participants (30.6\%) allowed an interval less than 5 minutes between eye drop and ointment, while $30.5 \%$ said they allowed an interval of 5-10 minutes. On the other hand, $27.8 \%$ of the participants indicated that they did not usually determine the interval between ointment and eye drop applications. Furthermore, $32.5 \%$ of participants indicated that they always 
washed their hands prior to administering eye drops, while $29.6 \%$ of the participants washed their hands occasionally. On the other hand, $37.8 \%$ of the study participants never washed their hands. These results are shown in Table 2.

Table 1: Demographic characteristics of the participants

\begin{tabular}{lcc}
\hline Characteristics & Frequency & Percentage (\%) \\
\hline Age (years) & & \\
$18-35$ & 455 & 63.3 \\
$35-50$ & 169 & 23.5 \\
$>50$ & 56 & 7.8 \\
Sex & & \\
Male & 229 & 31.8 \\
Female & 491 & 68.2 \\
Region & & \\
Central & 483 & 67.2 \\
Western & 83 & 11.5 \\
Eastern & 66 & 9.2 \\
Southern & 71 & 9.9 \\
Education level & & \\
College & 578 & 80.3 \\
High school & 111 & 15.4 \\
\hline
\end{tabular}

Table 2: Techniques used in the administration of eye drops.

\begin{tabular}{lcc}
\hline $\begin{array}{l}\text { Technique used in } \\
\text { administration of } \\
\text { eye drops }\end{array}$ & Frequency & Percentage (\%) \\
\hline $\begin{array}{l}\text { Eye contact with } \\
\text { bottle tip }\end{array}$ & & \\
Always avoided & 594 & 82.5 \\
$\begin{array}{l}\text { Occasionally avoided } \\
\text { Using both eye }\end{array}$ & 99 & 13.8 \\
drop and ointments & & \\
Eye drop applied first & 718 & 90.8 \\
Eye ointment applied & 46 & 6.4 \\
first & & \\
Time interval & & \\
between eye drop & & \\
and ointment & & \\
administration & 220 & 30.6 \\
<5 min & 25 & 30.5 \\
5-10 min & 200 & 27.8 \\
Undeternined interval & 200 \\
No interval (applied & 80 & 11.1 \\
at the same time) & & \\
Hand wash & 234 & 32.5 \\
Always & 213 & 29.6 \\
Occasionally & 272 & 37.8 \\
Never & \\
\hline
\end{tabular}

\section{Role of pharmacists}

It was revealed by 320 participants $(44.4 \%)$ that dispensing pharmacists gave instructions only on the number of eye drops, while 232 participants $(32.3 \%)$ reported that pharmacists gave instructions on the number of eye drops and administration techniques that should be used.

\section{Storage and disposal of eye drops}

Less than half $(40.9 \%)$ of the participants indicated that they kept their eye drops at the refrigerator door, while $42.7 \%$ said they stored the eye drops in warm places such as the bedroom and kitchen.

Regarding the expiration of the eye drops, 44.9 $\%$ ) reported that the eye drops had one month or less to expiry date. On the other hand, $41.7 \%$ of participants did not know the expiry dates of their eye drops.

Regarding the disposal of eye drops, most of the participants $(82.6 \%)$ reported that they disposed of the eye drops immediately after expiry. Moreover, almost all the participants (95.5\%) indicated that on expiry, they threw the eye drops in the garbage bin. These data are shown in Table 3.

Table 3: Storage and disposal of eye drops

\begin{tabular}{|c|c|c|}
\hline $\begin{array}{lll}\begin{array}{l}\text { Storage } \\
\text { disposal of } \\
\text { drops }\end{array} & \begin{array}{l}\text { and } \\
\text { eye }\end{array} \\
\end{array}$ & Frequency & Percentage (\%) \\
\hline \multicolumn{3}{|l|}{$\begin{array}{l}\text { Storage of eye } \\
\text { drops }\end{array}$} \\
\hline Inside the refrigerator & 87 & 12.1 \\
\hline At refrigerator door & 294 & 40.9 \\
\hline \multicolumn{3}{|l|}{$\begin{array}{l}\text { Expiry dates of eye } \\
\text { drops }\end{array}$} \\
\hline 1 month or less & 323 & 44.9 \\
\hline 1 week or less & 96 & 13.4 \\
\hline No idea & 300 & 41.7 \\
\hline \multicolumn{3}{|l|}{$\begin{array}{l}\text { Disposal of eye } \\
\text { drops immediately } \\
\text { after expiry }\end{array}$} \\
\hline Yes & 549 & 82.6 \\
\hline No & 125 & 17.4 \\
\hline $\begin{array}{l}\text { Disposal method } \\
\text { used for eye drops }\end{array}$ & & \\
\hline $\begin{array}{l}\text { Throw eye drops in } \\
\text { the garbage bin }\end{array}$ & 687 & 95.5 \\
\hline
\end{tabular}

\section{DISCUSSION}

It has been reported that failure on the part of patients to adhere to eye drop regimen may contribute to poor prognosis of their ophthalmic conditions and the exacerbation of symptoms. It is noteworthy that $80 \%$ of the patients administer their eye drops while sitting, standing, or in front of a mirror $[13,21]$. Surveys on the appropriate use of eye drops are essential because they provide data that enable researchers evaluate the overall understanding of the best technique to use, and to assess the contribution of pharmacists [20]. However, no specific technique 
has been found to be optimal, which indicates that instructions should be tailored based on the need of the patient [1]. It is important that ophthalmic medication is given the same priority as medications that are administered systemically. This underlines the fact that appropriate storage of medications is essential. When stored incorrectly, many drugs undergo physical and chemical changes which may result in loss of their potencies [2].

When pharmacists dispense ophthalmic drops, they should demonstrate their correct use to the patients. This ensures that future applications of the medication will be done correctly using the proper technique. Many patients forget to administer eye drops at the right time, while others neglect to administer the medication altogether [13]. In this study, majority of the participants reported that they received no form of instructions from their pharmacists. However, it should be noted that although instructions on how to instil ophthalmic drops are essential, it is not likely that all patients would follow these instructions accordingly [14]. Indeed, it has been reported that a good percentage of those instructed may not recall the exact details of the instructions given [15]. Ultimately, several visits may be required for some patients to achieve the best outcomes [11]. Generally, instructions help to minimise the risks and optimise the potential benefits.

Several studies have shown that $15-52 \%$ of ophthalmic patients touched the tip of the eye drop container to their eyes while applying the eye drops $[11,13,19,23]$. However, once instructions and corrections were received, the number of patients that touch their eyes with the tip of the eye drop container reduced significantly to $34 \%$ [11]. Similarly, evidence suggest that instructions on the desirability of washing the hands prior to eye drop application may significantly increase this practice [12]. However, evidence also suggest that the number of eye drops administered overall (and the length of time between applications) may not change appreciably even after instructions [12]. Factors associated with poor administration technique include old age, poor vision, lower educational level, lack of supervision, low adherence, and a history of instruction [13,15-17]. Ultimately, these factors may create several barriers such as leakage of drops, missing the desired spot for drops, contamination, and use of inadequate force to release the drops [18].

Another crucial issue about ophthalmic drops is the question of storage. In the current study, 40.9 $\%$ of the participants stored their eye drops at room temperature, and $30 \%$ of this group stored their drops in a relatively warm place (kitchen). This is consistent with the findings from other studies, which reported that most patients keep their eye drops in their bedrooms, bathroom, or kitchen [22,23]. Although room temperature varies significantly from one bedroom to another based on the country in which a patient lives, it is almost universally the case that storing eye drops in the bathroom can increase the chances of contamination. Furthermore, the kitchen is not the best place for eye drop storage in view of the presence vapours, volatile oils, and fluctuating (often high) temperatures, all of which have negative impact on the drugs. Generally, it should be noted that the storage conditions of ophthalmic preparations vary based on manufacturer's recommendations. While some should be refrigerated, others may be kept safely at room temperature. This variability has created confusion among patients, which further emphasises the need for clear instructions and follow-up sessions.

Most guidelines advocate that in hospital settings, eye drops should be discarded after seven days and replaced if the treatment continues. However, in non-hospital settings, eye drop bottles should be replaced every 28 days. It is not uncommon, however, to find expired eye drops in domestic medicine cabinets [24,25]. In this study, it was found that $41.7 \%$ of the participants continued to use their eye drops more than 28 days after opening them, irrespective of the expiry dates. This is indicative of the fact that most patients kept their eye drops in their medicine cabinets even after they had completed their course of treatment, without being aware of their expiry dates. Other studies have demonstrated that $68 \%$ of patients discarded the eye drops more than 28 days after they had been opened. This problem can be solved either during dispensing or during followup visits [12].

\section{CONCLUSION}

While most of study participants have a good knowledge of the right way to administer eye drops, some of the participants are unaware correct way of storage and disposal of eye drops. Therefore, there is a need for pharmacist to spend more time to counsel patients on this.

\section{DECLARATIONS}

\section{Conflict of Interest}

No conflict of interest associated with this work. 


\section{Contribution of Authors}

The authors declare that this work was done by the authors named in this article and all liabilities pertaining to claims relating to the content of this article will be borne by them.

\section{REFERENCES}

1. Waterman H, Evans JR, Gray TA, Henson D, Harper $R$. Interventions for improving adherence to ocular hypotensive therapy. Cochrane Database of Systematic Reviews 2013; (4): 1-82. Available from: doi:10.1002/14651858.CD006132.pub3.

2. Patel A. Ocular drug delivery systems: An overview. World Journal of Pharmacology 2013; 2(2): 47. Available from: doi:10.5497/wjp.v2.i2.47.

3. Shaw M. How to administer eye drops and eye ointment. Nursing Standard 2016; 30(39): 34- 36. Available from: doi:10.7748/ns.30.39.34.s42.

4. Gaudana R, Jwala J, Boddu S, Mitra A. Recent Perspectives in Ocular Drug Delivery. Pharmaceutical Research 2008; 26(5): 1197-1216. Available from: doi: 10.1007/s11095-008-9694-0.

5. Institute for Quality and Efficiency in Health Care (IQWiG). Using eye drops properly. [Internet] 2016. Available

from: https://www.ncbi.nlm.nih.gov/pubmedhealth/PMH00725 10/ [accessed 22nd October 2016].

6. Kass MA, Hodapp E, Gordon M, Kolker AE, Goldberg I. Part I. Patient administration of eyedrops: interview. Ann Ophthalmol 1982; 14(8): 775-9.

7. An JA, Kasner O, Samek DA, Lévesque V. Evaluation of eye drop administration by inexperienced patients after cataract surgery. Journal of Cataract \& Refractive Surgery 2014; 40(11): 1857-1861. Available from: doi:10.1016/j.jcrs.2014.02.037.

8. Mohindroo C, Ichhpujani P, Kumar S. How 'Drug Aware' are our Glaucoma Patients? J Curr Glaucoma Pract. 2015; 9(2): 33-37. Available from: doi:10.5005/jpjournals-10008-1181.

9. https://www.nps.org.au/australian-prescriber/articles/onthe-correct-use-of-eye-drops\#authors

10. htpp://www.safemedication.com/safemed.MedicationTips Tools/HowtoAdminister/HowtoUseEysDropsProperly. as px

11. Gupta R, Tinwala S, Singh H, Dave A, Wadhwani M, Dada T. To Study the Effect of Instructions on Improving the Eye Drop Instillation Technique in Patients With Primary Adult Onset Glaucoma. Investigative Ophthalmology \& Visual Science 2012; 53(14).

12. McVeigh KA, Vakros G. The eye drop chart: a pilot study for improving administration of and compliance with topical treatments in glaucoma patients. Clin Ophthalmol 2015; 9: 813-819. Available from: doi:10.2147 /OPTH.S82909.
13. Tatham AJ, Sarodia U, Gatrad F, Awan A. Eye drop instillation technique in patients with glaucoma. Eye 2013; 27: 1293-1298. Available from: doi:10.1038/eye.2013.187.

14. Al-Busaidi A, Samek DA, Oscar Kasner. Eye drop administration in patients attending and not attending a glaucoma education center. Oman J Ophthalmol 2016; 9(1): 11-16. Available from: doi:10.4103/0974$620 \times .176094$

15. Kholdebarin $R$, Campbell RJ, Jin YP, Buys $Y M$. Multicenter study of compliance and drop administration in glaucoma. Can J Ophthalmol. 2008; 43: 454-461. Available from: doi: 10.1139/i08-076.

16. Dietlein TS, Jordan JF, Lüke C, Schild A, Dinslage S, Krieglstein GK. Self-application of single-use eyedrop containers in an elderly population: comparisons with standard eye drop bottle and with younger patients. Acta Ophthalmol. 2008; 86: 856-859. Available from: doi:10.1111/j.1755-3768.2007.01155.x.

17. Newman-Casey PA, Dayno M, Robin AL. Systematic Review of Educational Interventions to Improve Glaucoma Medication Adherence: an update in 2015. Expert Rev Ophthalmol. 2016; 11(1): 5-20.

18. Davies I, Williams AM, Muir KW. Aids for eye drop administration. Survey of Ophthalmology 2017; 62(3): 332-345. Available from: doi:10.1016/j.survop hthal.2016.12.009.

19. Schwartz GF, Hollander DA, Williams JM. Evaluation of eye drop administration technique in patients with glaucoma or ocular hypertension. Curr Med Res Opin. 2013; 29(11): 1515-22. Available from: doi:10.1185/03007995.2013.833898.

20. Stone JL, Robin AL, Novack GD, Covert DW, Cagle GD. An objective evaluation of eyedrop instillation in patients with glaucoma. Arch Ophthalmol. 2009; 127(6): 732-6. doi:10.1001/archophthalmol.2009.96.

21. Strungaru MH, Peck J, Compeau EC, Trope GE, Buys YM. Mirror-hat device as a drop delivery aid: a pilot study. Can J Ophthalmol. 2014; 49(4): 333-8. Available from: doi:10.1016/j.jcjo.2014.04.012.

22. Tsai T, Robin AL, Smith JP. An evaluation of how glaucoma patients use topical medications: a pilot study. Trans Am Ophthalmol Soc. 2007; 105: 29-35.

23. Arunkumar S, Banagar B, Chanda. Eye drop instillation, a skill to be taught? Journal of Educational Research \& Medical Teacher 2014; 2(2): 20-22.

24. Gupta N, Alam A, Bhardwaj, Amin F. Prospective survey study on assessment and education of home medicine cabinet in general population of community. International Journal of Pharmaceutical Sciences \& Research 2011: 1237-1243.

25. Vuong T, Marriot JL. Unnecessary medicines stored in homes of patients at risk of medication misadventure. Journal of Pharmacy Practice \& Research 2006; 36(1): 16-20. Available from: doi:10.1002/j.20552335.2006.tb00879.x 